\title{
MOTIVATIONAL FACTORS IN APHASIA THERAPY: RESEARCH SUGGESTIONS
}

\author{
MERTON A. SHILL \\ Children's Psychiatric Hospital, University of Michigan, 1275 N. Hospital Dr., Ann Arbor, Michi- \\ gan 48109.
}

\begin{abstract}
Following Wepman (1953), the process of recovery from aphasia is seen as involving stimulation, motivation, and facilitation. The most adequate studies of aphasia therapy outcome focus on facilitation to the virtual exclusion of stimulation and motivation, although clinical experience suggests their importance. Motivation includes both the subjective and experiential aspects of the patient's rcsponse to aphasic disablement (anxiety, feelings of inadequacy) and the quality of the speech-therapist/ patient alliance during the treatment. Retrospective studies utilizing sufficiently improved aphasics and ratings of the patient-therapist alliance are suggested as preliminary means of defining "motivation " in this context more accurately. Research approaches are also outlined which would assess the importance of and interaction between the three factors implicated in recovery for therapeutic outcome.
\end{abstract}

Research into the outcome of aphasia therapy suffers from a variety of conceptual and methodological shortcomings (Darley, 1975; Smith, 1972). Darley (1977) notes:

No investigation to date has included an adequately selected control group of untreated patients for comparison with an adequate experimental group of treated patients. No study to date has been of such magnitude or scientific rigor as to yield unequivocal differential statements about the objectively measured improvement of patients with aphasia of various etiologies, severity and duration; site and extent of the lesion; and ages, health, and backgrounds of patients subjected to competent treatment regimens of various types, intensities, and durations.

Clinical experience and discussions of aphasia therapy repeatedly emphasize the importance of psychological factors, especially the role of motivation in the patient's treatment (Darlcy, 1972, 1975; Eisenson, 1949; Jenkins et al., 1975; Luria, 1963; Wepman, 1951, 1953). Reviews of research into the efficacy of aphasia therapy (e.g., Darley, 1975; Smith, 1972) provide no detailed guide, however, as to the assessment of motivational factors in treatment outcome research and the most sophisticated outcome studies to date have not dealt with them (e.g., Smith, 1972; Wertz et al., 1978). The inclusion of motivational

Address correspondence to: Merton A. Shill, Children's Psychiatric Hospital, University of Michigan, 1275 N. Hospital Drive, Ann Arbor, MI 48109. 
variables in studies of the efficacy of aphasia therapy is needed to bring research strategy into line with clinical experience.

\section{The Recovery Process in Aphasia}

Wepman (1953) has suggested that the various processes involved in recovery from aphasia are: 1) stimulation - what is done to and with the patient by any external agency; 2) facilitation - what the impaired nervous system is capable of doing; 3) motivation - what the state of the internalized drive of the patient might be.

Stimulation here refers to the therapeutic stimulus input, which elicits language response from the patient in an attempt to rehabilitate language functions. Facilitation is the physiological capacity of the organism to improve or alter its cortical functioning so as to permit new language integrations.

The main focus of this paper is motivation, the third aspect of the recovery process according to Wepman. Motivation is the "psychological state of readiness [which] must . . . exist before maximal learning of the formation of new, operative neural integrations are possible" (Wepman, 1953). This state of readiness is the willingness to strive for the rehabilitation of one's language capacity.

Although motivation will be discussed further below, this tripartite conception of the recovery process underlies the approach of this paper.

While there seem to be numerous nonsystematic, ad hoc treatment approaches to aphasia, two main schools of thought have emerged: the stimulation approach and the programmed instruction approach (Darley, 1975). The former is said to rely on intensive stimulation of the patient to produce the cortical integrations necessary for language, while the latter is described as an educational process, based on operant conditioning methods evolved by learning theorists (e.g., Holland and Sonderman, 1974; Sarno and Sands, 1970).

This description of the two approaches suggests that the learning theory methods do not rely on neural integration even though this is not specified. Learning theorists typically do not discuss the cortical mechanisms responsible for learning, although some have been suggested, e.g. neurobiotaxis (Hebb, 1949). The usual focus of learning theory is upon the observables of behavior so as to avoid speculation about mental functioning or basic cortical processes. Since learning is at least partially the result of a neurophysiological process, it must involve cortical integrations. It seems impossible then to describe recovery from aphasia by programmed instruction methods without assuming the occurrence of some nonobservable neural change that permits the new language skills to be learned, stored in memory, and reproduced (cf. Luria, 1970; Wepman, 1953).

In his definition of stimulation, Wepman (1953) emphasized the role of external persuasion by those in the patient's environment to provide stimuli to which he may react. Both the so-called stimulation techniques, as well as programmed 
instruction, seem therefore to involve the stimulation initiated by an external source of several processes involved in the multimodality reception, understanding, and expression of language without the direct administration of any biochemical agent (cf. Jenkins et al., 1975; Luria, 1963, 1970; Schuell, 1974). The basic mechanism involved in both approaches is a language procedure that has a remedial impact upon neural function (Jenkins et al., 1975; Luria, 1963, 1970). The nature of and process underlying this mechanism are at present unknown but its presence is assumed from the gains in language facility of treated aphasic patients (Smith, 1972; Wertz et al., 1978).

\section{Treatment Outcome Research}

A guiding theoretical conception as to the nature of the process of recovery from aphasia could serve to focus treatment outcome research on the most relevant variables involved. The circularity in this suggestion is only apparent. A theoretical conception of the rccovery process is a hypothesis which could be corrected in the light of research results. It need not rigidly presuppose knowledge of the very factors being researched.

Current studies of treatment outcomes suggest only limited attention to selected aspects of the recovery process, however described. Facilitation is the most frequent focus in speech therapy research. Accordingly, many studies monitor at least some of the following factors: age of the patient, etiology and character of aphasia, the site and extent of the lesion and associated defect, e.g., oral apraxia, sensory and motor deficits (Darley, 1975; Wertz et al., 1978). By contrast, the roles of stimulation and motivation have gone largely unheeded. The intensity of the therapy, the competence of the clinicians, and the patient's social milieu, all bear upon the amount of appropriate stimulation he receives. The empathy and support provided by the clinician, the patient's nonlanguage behavior characteristics, and possibly the time between morbid onset and the start of therapy, could influence the patient's motivation (cf. Eisenson, 1949).

Moreover, stimulation, facilitation, and motivation are intimately related (Wepman, 1953). It follows that research into treatment outcome should reflect this by exploring the interactions between the variables indexing stimulation, facilitation, and motivation. No study to date has done so.

More research attention to the stimulational and motivational aspects of speech therapy could provide an empirical foundation for the following description of treatment, which is essentially a considered clinical judgement, in need of testing:

Therapy is organized, goal directed stimulation based upon a rccognition of the patient's needs, his drives, his motivation. As stimulation is provided at a time when the organism is capable of response it tends to facilitate neural integrations. If the direction of the stimulation at the proper time is in keeping with the 
patient's psychological state of readiness, if the stimulation is within the modality of language which meets the patient's needs, if the stimulation provides the end reward by realizable and recognizable goals of achievement, then success in therapy is more likely to follow. (Wepman, 1953)

\section{Psychological Aspects of Treatment: Motivation}

Clinical experience shows that mere stimulation of a neural system capable of facilitation is not enough to produce recovery from aphasia (Wepman, 1953). His motivational state dictates the level of goal-directed behavior of which the patient is capable (Luria, 1970).

Discussions of motivation and its importance for treatment success by the leading authorities tend to be sparse, although its importance is repeatedly stressed. Jenkins et al. (1975), in the revised edition of Schuell's Aphasia in Adults, do not discuss motivation per se, but pay considerable attention to the clinician's need for empathy. Their discussion assumes the importance of motivation and a good treatment alliance for progress in therapy (cf. Darley, 1975). Eisenson $(1949,1973)$ suggests that the persistence of euphoria after a patient has had sufficient time to realize the amount of his linguistic disturbance indicates a wish to avoid dealing with his language handicap. After such realization, Eisenson suggests that motivation can be assumed to be present and need not be focused on until the patient reaches a plateau in improvement. Now the patient may have to be urged to make the necessary effort (Eisenson, 1973).

Although he seems to distinguish between motivation and level of aspiration, Eisenson's discussion of the latter is a useful addition to the conception of the role of motivation. He suggests that the therapist help the patient adjust to a realistic, short-term level of aspiration, thus avoiding unattainably high objectives. On the other hand, if the patient's aspiration level is too low, this may indicate a fear of failure, and the therapist should help the patient accept failure as a necessary part of the treatment and the growth process (Eisenson, 1973). Patients improve and respond positively to encouraging instructions, and do significantly more poorly on language tasks when discouraged (Stoicheff, 1960). The difficulty of the specific language goal the patient attempts to attain can affect his willingness to work towards its realization.

Although Luria conceives of treatment as the "restoration of brain function," he emphasizes the role of motivation $(1963,1970)$. The recovery or restoration process after trauma involves the deinhibition of functions temporarily depressed by the trauma, the substitute use of the other hemisphere and/or functional reorganization. It involves an "active conscious activity" towards compensation of the defect (Luria, 1963) which is due to the functional dissociation of speech from the mainstream of conscious behavior (Luria, 1970). Despite his marked neurological approach to aphasia, Luria emphasizes the importance of motivational factors in aphasia rehabilitation. 
The role and conception of motivation in the treatment of aphasics has been emphasized most by Wepman, and the above review of leading authorities does not add significantly to his original conceptions (Wepman, 1951, 1953), with the exception of Eisenson's use of the concept of aspiration level. It is difficult to go beyond the prevailing vague description of motivation. Clearly, it is an inferential notion based on behavioral observations. For this reason a more detailed definition of motivation will not be offered here. It seems more advisable to adopt an empirical approach to this question and to pursue pilot research by sampling the motivational domain relevant to aphasia in order to produce an appropriate definition. Some preliminary research suggestions in this direction are made below.

Due to the interaction of stimulation and facilitation with motivation in determining treatment outcome, the former could be decisive in limiting the effects of motivation on the rate and ultimate level of recovery. No matter how strongly motivated, a patient with extensive, bilateral lesions would most probably have a poorer prognosis for treatment outcome than one with only mild unilateral damage (Smith, 1972). Without adequate motivation little progress in treatment could, of course, be expected. But within limits a patient's high motivational level may cause him to exceed the expected improvement range based on an assessment of his language impairment alone. The impact of motivation on ireatment outcome cannot, therefore, be assessed adequately without a concurrent consideration of stimulation and facilitation. This interactive approach to treatment outcome research provides the clearest means of isolating and possibly weighting the different factors responsible for progress in treatment.

The factors affecting the patient's motivational state are varied and some are probably interrelated although the precise relationships involved are as yet unknown. Wepman (1951) identified 34 nonlanguage characteristics of aphasics which ought to direct the attention of the clinician to a full consideration of the patient's personality and the appearance of behavioral aberrations. These posttraumatic behavioral manifestations seem to be due to both the effects of the lesion and the patient's attempts to regain his ego integrity and struggle with the disorganizing effects of the trauma. The respective individual and reciprocal contributions of these two factors are highly relevant but seem impossible to determine at present.

Some of the characteristics which Wepman (1951) mentions have significant motivational implications, e.g., the loss of attention and concentration, feelings of inadequacy, reduced initiative, and anxiety. Different intensities of these characteristics are important too. Anxiety bordering on panic (cf. Goldstein, 1942) would inhibit progress, whereas no anxiety, if evidencing a lack of concern about one's disabled speech, would foreclose rehabilitation. Presumably, an optimal level of anxiety would operate to motivate the patient sufficiently to want to overcome his handicap short of immobilizing him with fright. The degree of 
anxiety can be affected by what the patient understands about his disability, his expectations of recovering his speech, the acceptance he receives from his family and friends, and the degree to which the explicit and implicit demands of all those treating his disability are encouraging, critical, or disheartening (Darley, 1975).

The importance of the patient's motivation can perhaps be emphasized and appreciated from the impressions of both clinicians and recovered patients as to their feelings soon after onset. Few attempts have been made to obtain patient descriptions of their reactions to cerebral injury and speech impairment. Wepman (1951) obtained introspective reports from over 50 aphasic patients of their experiences after trauma due to war injuries. Amnesia in the early post-traumatic periods sometimes limited the range of the reports, but other patients were conscious almost immediataly after trauma. The descriptions of the early post traumatic period conveyed the impression of a vegetative existence, devoid of anxiety, in which basic bodily needs were cared for by others. The earliest attempts at communication were directed at obtaining need satisfaction and significantly, from the point of view of motivation, no memory for previous communication ability existed. Even under questioning by professional staff these patients reported no sense of loss at their inability to speak. They became accommodated to their daily routines and the need to improve was only rarely felt.

The first focus of concern was not their speech disability but their recognition of the loss of the use of a limb. When visited by family members who expressed concern for their condition, these patients reported almost unanimously that even then their inability to communicate with others was of little importance. At no stage was there any strong concern about the need to overcome their speech loss.

The picture revealed by these traumatic aphasic patients is very similar to the descriptions of the reactions to aphasia onset given by some CVA victims. Moss (1972) reports that his own and the reactions of others to the loss of speech generally did not produce anxiety. Significantly, in the few cases in which it did, the patient was either impelled by family or internal persuasion immediately to begin the rehabilitation process, sometimes with startling success.

These accounts are largely unsystematic and deal with discrete etiological entities, but they require further systematic attention since they suggest that it is partly the responsibility of those in the patient's milieu to "goad him into action," to make him sufficiently dissatisfied with his speech impediment to want to overcome it. Individual differences no doubt exist, as well as differences based on etiology of the aphasia.

The early apathy and confusion are the immediate result of the trauma in some cases. In others such confusion or lack of attention may be indicative of anosognosia and may even index a severe personality disturbance or psychosis (Weinstein and Lyerly, 1976). In such cases, however, the problem is more complex, and is related to premorbid personality patterns and involves more than merely motivational handicaps (Weinstein and Lyerly, 1976). 
The gathering conviction of the importance of starting treatment as soon as possible or at the optimal moment to maximize its likelihood of success (e.g., Butfield and Zangwell, 1946; Smith, 1972; Vignolo, 1964; Wertz et al., 1978) suggests that external motivation of the patient should begin as early as can be done appropriately and sensitively post-onset, so as to ensure the best opportunity for progress in treatment. As a result of his obtaining these patient reports, Wepman related his clinical impression that even the understanding the patients obtained thereby enhanced the ultimate recovery level. The attempt of the clinician therefore to motivate the patient and the date post-onset when the patient is judged to respond to this seem very important to consider in researching aphasia treatment outcome.

Given the clear relevance of patient reports of their experiences in treatment, it would seem helpful to study the reactions of sufficiently recovered aphasic patients systematically so that a more precise understanding of all treatment-related and extra-treatment variables could be acquired. This could greatly enhance the study of the efficacy of treatment if proper controls were administered for other important variables, e.g. the site and etiology of the lesion, character of the aphasia, the age of the patient, etc. (cf. Wertz et al., 1978).

The only study of the reactions of aphasic patients to their treatment I have been able to locate is reported by Skelly (1975). Fifty stroke patients classified as severely impaired on admission and who had recovered sufficient speech were asked structured and open-ended questions about their inpatient treatment. No details as to the nature of their aphasias are supplied in the report. A content analysis of these patient interviews uncovered three areas of unanimous or serious concern relevant to patient motivation: i) Ability to comprehend-The patients reported the ability to understand what was said in their presence much sooner than their professional helpers would have expected. Unfortunately, they were often traumatized by what they heard, feeling dehumanized by the impersonal clinical discussions of their condition. Coupled with the lack of an explanation for their symptoms, they experienced heightened fear and anguish. ii) Nonverbal communication-Patients reported marked awareness of the nonverbal reactions of hospital personnel and family members to their impairment, especially impatience. They felt that these reactions adversely affected their morale, motivation, and progress. iii) Need for information-The patients wanted to know the reasons for various interventions, often not given because the professional staff assumed that they were incapable of understanding. (Psychologically, this may be seen as a way of mastering the anxiety that these patients felt about their predicament.) iv) Respect for personhood-Invasions of patient privacy, from intrusive questions about finances through requests to "show off" their achievements in treatment, were considered undignified.

While some of the feelings these patients reported might be so serious as to warrant psychotherapy in order to render speech treatment more effective, their impact within the patient's speech-treatment milieu on his progress seems highly 
relevant. Research into the outcome of aphasia treatment which does not take account of these factors could attribute success or failure to a particular treatment mode or intensity; for instance, while these motivational factors could have been quite decisive, influenced positively or negatively by someone in the patient's environment other than the speech therapist, e.g. another member of the treatment team (Moss, 1972).

The attempts of the patient at covert or private rehearsal of speech production-"self-training" (Darley, 1972)—seem also to reflect motivation. Given adequate patient understanding and possible appropriate assistance from family or staff, the frequency and duration of a patient's attempts to recover speech independently may be an important index of personal motivation, although it would also affect the level of stimulation and be a function of the post-morbid condition of the brain (facilitation).

Although not accounted for as a function of motivation, the report of successes of programmed instruction therapy with those patients who do not respond to the more traditional speech therapies (cf. Darley, 1975) is achieved possibly because the stepwise, goal-attainment procedure involved gives clear and immediate reinforcement to the patient's efforts, thus serving a motivational purpose (cf. Sarno and Sands, 1970; Luria, 1970).

While all the factors discussed have motivational relevance for the aphasic patient, their assessment and control in a research study on treatment outcome present considerable difficulty. Although highly sophisticated methods are not presently available, the following suggestions are intended as interim practical measures of the observable aspects of behavior relevant to the patient's motivation.

As a general guide, content coding of interviews with successfully treated aphasic patients could be conducted in order to determine which motivational factors were most successful in treatment. This procedure would assist in a more systematic definition of the domain of motivation. The following are also suggested in the light of the above discussion: Clinical ratings of the degree of nonlanguage characteristics and aberrations mentioned by Wepman (1951); home observational visits (cf. Bermann, 1970) to obtain a general picture of family dynamics and acceptance of the patient as well as specific help given in language rehearsal; clear specification of treatment goals even if these are only very modest and on a short-term, day-to-day basis; the number and intensity of private rehearsal attempts made by the patient; a clinical rating of the attempts of the therapist to develop a goal striving in the patient; time post-onset of the patient's ostensible attempts to talk; a record of the number of therapy appointments missed; and the amount and nature of any psychotherapy, physiotherapy, and other treatment regimes the patient received in addition to speech therapy.

By this means, a motivational matrix or check list could be developed to assist in monitoring and scoring the patients motivation so as to assess its impact on treatment progress. 


\section{Patient-Therapist Relationship}

This seems to involve both stimulation and motivation aspects as the description of the role of the therapist from Darley (1975) indicates:

The clinician therapist continually provides information, insight, encouragement, and optimistic effort; he assuages tendencies to self-criticism, selfpunishment, anxiety and despair. By his supportive, nonprovocative manner and his systematic schedule of language stimulation he conveys to the patient that the problem is understood and can be dealt with constructively . . . [this activity of the therapist] is an essential ingredient of the clinical situation and perhaps in some cases the only one that makes a great deal of difference.

Wepman (1951) designated these factors collectively as "climate" and also emphasized that many aphasic patients need physical care because of accompanying nonlanguage symptoms, e.g., hemiplegia or visual impairments. The therapist ought to be instrumental with others in facilitating the care and management of such difficulties as well as demonstrating a sincere understanding of the additional hardships they create.

The patient's over-dependence on the therapist (Eisenson, 1949) can also complicate the treatment process, especially if it produces resentment on the part of the therapist and prevents the patient from utilizing his existing resources to the fullest.

This multifaceted interpretation of the therapist-patient relationship is not reflected in current studies of recovery from aphasia.

Three main and interrelated aspects relating to the clinician's role seem to emerge from these descriptions: the therapist must foster the treatment alliance, he must be competent in the practice of language stimulation, and must enhance and help channel the patient's motivation towards language recovery. While stress on the therapist's competence highlights his role in the stimulation aspects of recovery from aphasia, all of these factors also serve to enhance the patient's motivation. The therapist's empathy, supportiveness, and encouragement tend to create confidence in him on the part of the patient and a willingness to cooperate in the therapy. The therapist's competence will also facilitate this by accurately conveying the sense that he knows what he is about. These motivational boosts are thus inextricably interwoven and are indispensible to maximizing the chances for therapeutic success.

Although this emphasis on the nonlanguage aspect of the clinician's role is hardly revelatory, some patients feel its neglect adversely affected their recovery from aphasia (Skelly, 1975). Notwithstanding the tenor of previous research studies therefore, the patient therapist relationship must be included in studies of treatment outcome.

If the holistic treatment approach is followed, thus not limiting the intervention to speech treatment alone, the factors that become involved in the treatment 
situation increase in complexity. The understanding of the patient in regard to his emotional, familial, social, speech and physical disabilities, so as to attempt his total rehabilitation, implies some assessment of the nature and impact of the measures taken to deal with all of these factors in any study of treatment outcome. This would therefore require assessment of the quality of the treatment alliance and the competence of the clinicians. With due regard to the patient's rights and sensitivities, therapy sessions could be videotaped or observed and the therapist-patient relationship rated on simple five- or seven-point scales by experienced clinicians applying preselected criteria, e.g. amount of empathy, quality of rapport established, etc. While a competent clinician would presumably be capable of fostering a good treatment relationship with the patient, other aspects of competence should also be assessed, e.g. amount of experience and training. The objective here is to begin the difficult process of systematic monitoring of those variables to which clinical experience and patient reaction assign great importance in order to assess their influence systematically on the outcome of aphasia therapy.

\section{Research Designs}

Current studies of aphasia therapy outcome do not generally monitor the various relevant independent variables adequately. These are site and extent of lesion, etiology, severity of the aphasia, duration of the disability, age at onset, general health factors, social class, patient's education, and type, intensity, and duration of treatment (but see Wertz et al., 1978). The factors indexing the patient's motivation and the quality of the therapeutic alliance already discussed should also be included.

Suggested designs for aphasia therapy outcome research are displayed in the tables. They reflect an attempt to monitor stimulation (e.g., therapy), facilitation (physiologic condition of the organism), and motivation, both concurrently and in interaction, and to judge their effect on treatment outcome.

Table 1 is a hypothetical data matrix which would be generated by a study of the effect of psychological factors and treatment types on a group of patients all homogeneous as to lesion characteristics (site, etiology, duration), severity of aphasia, general health factors, social class, education, etc. This design could have been adopted, for example, in the VAH cooperative study of aphasia (Wertz et al., 1978), where for the first time the outcome of individual or group treatment of patients homogeneous as to age, etiology, education, and the extent of disability, was studied. Such an approach would have permitted the assessment of the impact of various patterns of motivation and treatment alliance quality across treatment types, and answer the question: irrespective of the type of therapy offered, are the motivation of the patient and the quality of the treatment alliance relevant to treatment outcome? The design suggested here would also 


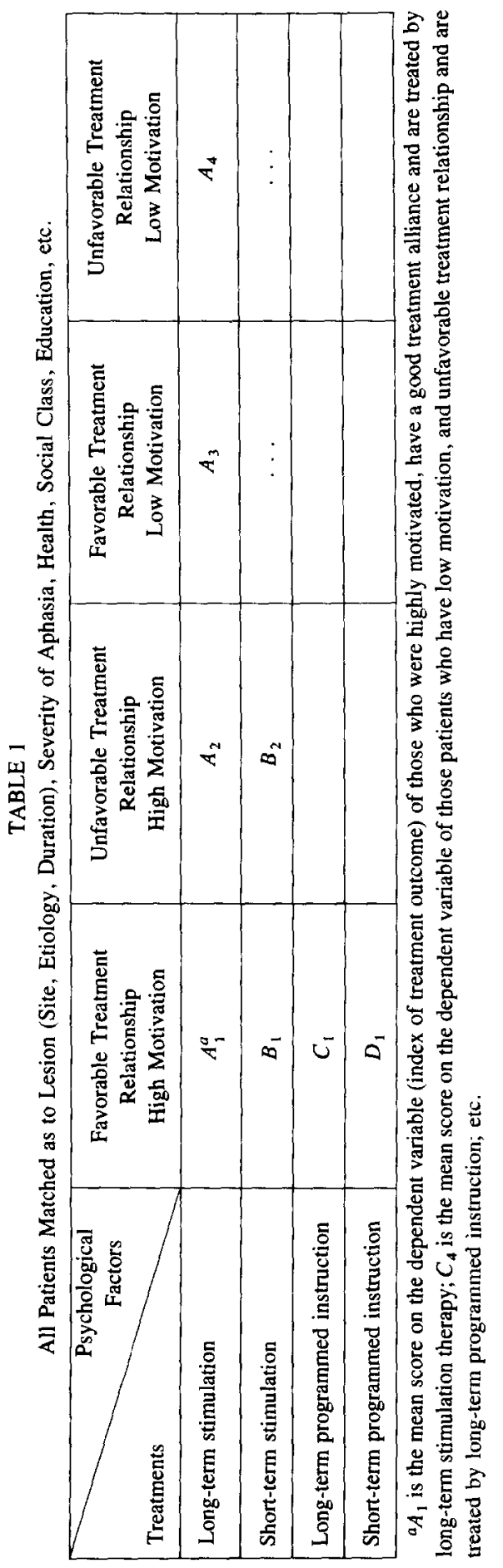




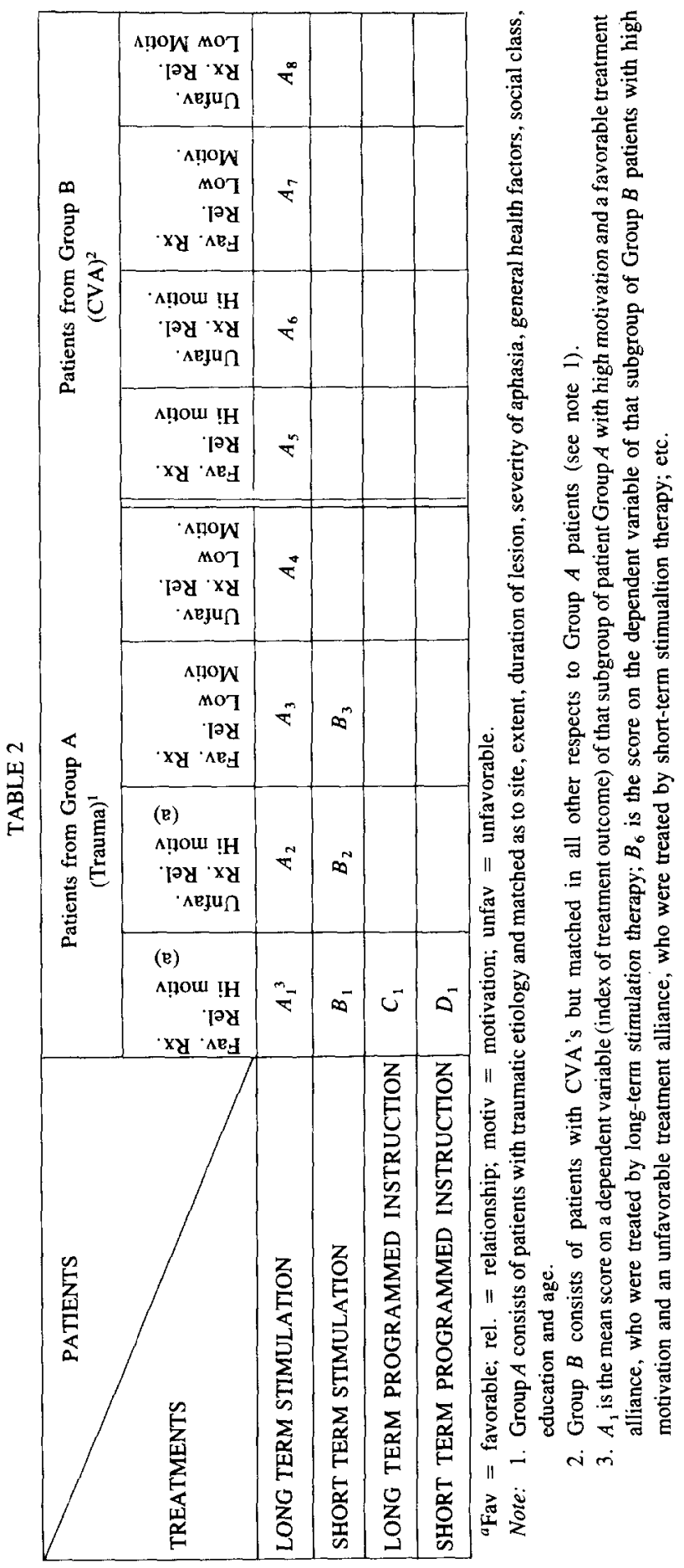


permit an answer to the parallel question: which treatment modalities provide the greatest therapeutic gains across all motivational and therapy alliance conditions? Most importantly, this research design would assess the interaction between psychological and treatment variables so as to be able to determine which combination of patient motivation and treatment alliance factors, together with the type of therapy, provides best treatment outcome. For example: Is the combination of an unfavorable treatment relationship and low motivation more detrimental to progress in long-term stimulation than long-term programmed instruction therapy? Does an unfavorable treatment relationship affect progress in group treatment to the same extent as in individual treatment since in the former, the relationships with other patients could be significant?

Different questions could be answered in a study based on the design in Table 2. This research approach would compare the impact of psychological treatment variables between two or more groups of patients with different etiology but matched in as many other respects as possible, e.g., site, extent and duration of lesion, severity of aphasia, general health factors, social class, education, and age but differing only as to traumatic vs acquired etiology (CVA) of aphasia. This design would provide answers to the following questions: 1) Do patients with traumatic ctiology requirc a particular combination of the therapeutic alliance and motivation in order to make progress in aphasia therapy, irrespective of treatment modality? 2) Do patients with acquired etiology (CVA) require a particular combination of the therapeutic alliance and motivation in order to make progress in aphasia therapy, irrespective of treatment modality? 3) Under which treatment regimen do patients with traumatic etiology fare best? 4) Under which treatment regimen do patients with acquired etiology fare best? 5) Which combination of patient motivation and treatment alliance variables with type of treatment modality provides the best results for patients with traumatic etiology? 6) Which combination of patient motivation and treatment alliance variables with type of treatment modality provide the best results for patients with acquired etiology?

A Table 2 design would also permit the comparison of therapy outcome between the two groups of patients, according to the nature of their etiology. A comparison of the relevant results would thus permit judgements as to the appropriate matching of etiological conditions, psychological factors, and treatment modalities to maximize the changes for optimal success in treatment.

Statistical analysis would be conducted according to 2-way factorial design analysis of variance (Hays, 1973).

\section{Conclusion}

This paper has emphasized certain facets of the psychological processes involved in treatment for aphasia and suggested some preliminary research strategies to assess their impact. Whatever treatment approach may be used with 
a particular patient, that patient's motivation and the quality of the therapist-patient relationship play a significant role in the recovery process which research studies of treatment outcome should no longer overlook.

The author gratefully acknowledges the advice and encouragement of Humberto Nagera, M.D., Chief of Youth Services, Department of Psychiatry, University of Michigan Medical Center, Ann Arbor, and Ann and Steven Zubrik in the preparation of this paper.

\section{References}

Bermann, E. (1970). Scapegoat. Ann Arbor: University of Michigan Press.

Butfield, E., and Zangwill, O. L. (1946). Reeducation in aphasia: A review of seventy cases. $J$. Neurol. Neurosurg. Psychiat. 9:75-79.

Darley, F. L. (1972). The efficacy of language rehabilitation in aphasia. J. Speech Hear. Dis. 37:3-21.

Darley, F. L. (1975). Treatment of acquired aphasia. In W. J. Friedlander (ed.), Advances in Neurology, Vol. 7. New York: Raven Press.

Darley, F. L. (1977). A retrospective view: Aphasia. J. Speech Hear. Dis. 42:161-169.

Eisenson, J. (1949). Prognostic factors related to language rehabilitation in aphasic patients. $J$. Speech Hear. Dis. 14:262-264.

Eisenson, J. (1973). Adult Aphasia: Assessment and Treatment. New York: Appleton.

Goldstein, K. (1942). Aftereffects of Brain Injuries in War: Their Evaluation and Treatment. New York: Grune and Stratton.

Hebb, D. O. (1949). Organization of Behavior. New York: Wiley.

Holland, A. L. and Sonderman, J. D. (1974). Effects of a program based on the token test for teaching comprehension skills to aphasics. J. Speech Hear. Res. 17:589-598.

Hays, W. L. (1973). Statistics for the Social Sciences (2nd ed.). New York: Holt, Rinehart \& Winston.

Jenkins, J. J., Jiminez-Pabon, E., Shaw, R. E., and Sefer, J. W. (1975). Schuell's Aphasia in Adults: Diagnosis, Prognosis and Treatment. Hagerstown, Md.: Harper \& Row.

Luria, A. R. (1970). Traumatic Aphasia. The Hague: Mouton.

Luria, A. R. (1973). Restoration of Function after Brain Injury. New York: MacMillan.

Moss, C. S. (1972). Recovery with Aphasia. The Aftermath of My Stroke. Urbana, Illinois: University of Illinois Press.

Sarno, M. T. and Sands, E. (1970). An objective method for the evaluation of speech therapy in aphasia. Arch. Phys. Med. Rehab. 51:49-54.

Schuell, H. (1974). Aphasia Theory and Therapy: Selected Lectures and Papers of Hildred Schuell. L. F. Sies (ed.). Baltimore: University Park Press.

Skelly, M. (1975). Aphasia patients talk back. American J. Nurs. 75:1140-1142.

Smith, A. (1972). Diagnosis, Intelligence, and Rehabilitation of Chronic Aphasics: Final Report. Department of Physical Medicine and Rehabilitation, University of Michigan, Ann Arbor, Michigan.

Stoicheff, M. L. (1960). Motivating instructions and language performance of dysphasic subjects. $J$. Speech Hear. Res. 3:75-85.

Vignolo, L. A. (1966). Evolution of aphasia and language rehabilitation: A retrospective exploratory study. Cortex 1:344-367.

Weinstein, E. A. and Lyerly, O. G. (1976). Personality factors in jargon aphasia. Cortex 12:122133. 
Wepman, J. M. (1951). Recovery from Aphasia. New York: Ronald.

Wepman, J. M. (1953). A conceptual model for the processes involved in recovery from aphasia. $J$. Speech Hear. Dis. 18:4-13.

Wertz, R. T., Collins, M., Weiss, D., Brookshire, R. H., Friden, T., Kurtzke, J. F., and Pierce, J. (1978). Veterans Administration cooperative study on aphasia: Preliminary report on a comparison of individual and group treatment. Presented to the American Association for the Advancement of Science, Washington, D.C., February, 1978. 